\title{
Deficiency of Functional Iron-Sulfur Domains in ABCE1 Inhibits the Proliferation and Migration of Lung Adenocarcinomas By Regulating the Biogenesis of Beta-Actin In Vitro
}

Qian Yu Xu Han Da-Li Tian

Department of Thoracic Surgery, The Fourth Affiliated Hospital of China Medical University, Liaoning, China

\section{Key Words}

Abce $1 \cdot \beta$-actin $\bullet$ Fe-S cluster $\bullet$ Lung adenocarcinoma $\cdot$ Migration

\begin{abstract}
Background/Aims: ATP-binding cassette transporter E1 (ABCE1), a unique ABC superfamily member that bears two Fe-S clusters, is essential for metastatic progression in lung cancer. Fe-S clusters within ABCE1 are crucial for ribosome dissociation and translation reinitiation; however, whether these clusters promote tumor proliferation and migration is unclear. Methods: The interaction between ABCE1 and $\beta$-actin was confirmed using GST pull-down. The lung adenocarcinoma (LUAD) cell line A549 was transduced with lentiviral packaging vectors overexpressing either wild-type $A B C E 1$ or $A B C E 1$ with Fe-S cluster deletions ( $\triangle A B C E 1$ ). The role of $\mathrm{Fe}-\mathrm{S}$ clusters in the viability and migration of cancer cells was evaluated using clonogenic, MTT, Transwell and wound healing assays. Cytoskeletal rearrangement was determined using immunofluorescent techniques. Results: Fe-S clusters were the key domains in $A B C E 1$ involved in binding to $\beta$-actin. The proliferative and migratory capacity increased in cells overexpressing ABCE1. However, the absence of Fe-S clusters reversed these effects. A549 cells overexpressing ABCE1 exhibited irregular morphology and increased levels of cytoskeletal polymerization as indicated by the immunofluorescence images. In contrast, cells expressing the Fe-S cluster deletion mutant presented opposing effects. Conclusion: These results demonstrate the indispensable role of Fe-S clusters when ABCE1 participates in the proliferation and migration of LUADs by interacting with $\beta$-actin. The Fe-S clusters of ABCE1 may be potential targets for the prevention of lung cancer metastasis.
\end{abstract}

\section{Introduction}

Lung cancer has the highest incidence of malignant tumor and is the leading cause of cancer mortality worldwide [1]. Lung adenocarcinoma (LUAD), a subtype of non-small 
cell lung cancer (NSCLC), is a major pathological type of cancer that manifests with diverse etiology, clinical symptoms and pathogenesis. Despite modern therapies, the prognosis of many patients with LUAD is poor due to the cancer's rapid progression, early metastasis and insensitivity to radiation, chemotherapy and epidermal growth factor receptor tyrosine kinase inhibitors (EGFR-TKIs) [2]. The developmental mechanisms of LUAD are complicated and poorly understood; therefore, identifying genes associated with LUAD and developing novel treatments to improve the prognosis of patients with LUAD are of paramount importance.

ATP-binding cassette transporter E1 (ABCE1), also known as RNase L inhibitor (RLI) or host protein (HP) 68, is a member of the ATP-binding cassette (ABC) protein superfamily [3]. ABCE1 has been reported to act as an RNase L inhibitor in the regulated 2-5A/RNase L pathway [4] and to participate in HIV-1 capsid assembly [5]. Post-termination ribosomes were split into large and small subunits by ABCE1 for participation in translation reinitiation [6].

Recently, emerging studies have reported increased ABCE1 expression in human tumors $[7,8]$. Furthermore, the levels of ABCE1 have been closely linked to clinical stages, which indicates that elevated ABCE1 expression may induce tumor proliferation and promote invasive behavior [9]. Therefore, improved treatments that reduce ABCE1 expression might inhibit tumor progression. Unlike other members of the ABC family, ABCE1 contains two nonequivalent diamagnetic [4Fe-4S $]^{2+}$ clusters (Fe-S clusters) at the $\mathrm{N}$-terminus [10]. This unique Fe-S cluster domain is essential for both ribosome binding and recycling to advance mRNA translation. An ATP-dependent conformational change within ABCE1 results in widening the Fe-S clusters into to a wider angle, which are required for binding of ABCE1 with the ribosome 30S subunit[11]. . Further study revealed that the Fe-S cluster domain rotates 160 degrees to anchor in the location between the S12 ribosomal protein and the rRNA of the small ribosomal subunit. When the ATP is hydrolyzed in the nucleotide-binding domains (NBDs), the FeS cluster domain rotates back, and ABCE1 dissociates from the small ribosomal subunit [12]. However, the specific functional relevance of the Fe-S clusters in ABCE1 regarding carcinogenesis remains unknown.

The actin cytoskeleton is the major structural network found in most eukaryotic cells $[13,14]$. Dynamic actin cytoskeleton remodeling modulated by G-protein coupled receptor protein signaling pathway is necessary during cancer metastasis regardless of the involved migratory organelle $[15,16,17]$. Shankar and colleagues indicated that a reduction in actinbased structure reassembly could reverse the morphological changes that are required for the migration and invasion of tumor cells [18]. They emphasized the importance of the actin cytoskeleton in the generation and development of tumors. Two isoforms, $\beta$-actin and $\gamma$-actin, have been reported in most non-muscle cell types as components of the cytoskeleton; $\beta$-actin has been confirmed to be responsible for cell adhesion and contraction [19]. Our team indicated a specific interaction between ABCE1 and $\beta$-actin in a previous study [20].

Thus, exploring the regulatory mechanisms associated with actin organization may provide new insights for targeting metastatic processes in cancer therapy. In this study, we investigated the correlation between the Fe-S clusters of ABCE1 and the $\beta$-actin cytoskeleton. We constructed lentiviral vectors containing sequences coding for wild-type ABCE1 and a mutant of ABCE1 with Fe-S cluster-specific deletions ( $\triangle \mathrm{ABCE} 1$ ). ABCE1 and $\triangle \mathrm{ABCE} 1$ were then overexpressed in the human LUAD cell line A549 to evaluate the role of ABCE1 Fe-S clusters in tumor cell proliferation and migration.

\section{Materials and Methods}

Cell lines and culture conditions

A549 human LUAD cells and 293T cells (originally from American Type Culture Collection, ATCC) were obtained from the Shanghai Biological Sciences Institute in China. A549 cells were cultured in F12K (Gibco, Grand Island, NY, USA) medium supplemented with 10\% fetal bovine serum (FBS, HyClone, Logan, UT, USA) 


\section{Cellular Physiology Cell Physiol Biochem 2017;44:554-566 \begin{tabular}{l|l|l}
\hline DOI: 10.1159/000485090 & $\begin{array}{l}\text { C } 2017 \text { The Author(s). Published by S. Karger AG, Basel } \\
\text { www.karger.com/cpb }\end{array}$
\end{tabular}}

Yu/Han/Tian: Fe-S Cluster of ABCE1 and Lung Adenocarcinoma Metastasis

at $37^{\circ} \mathrm{C}$ in a humidified atmosphere containing $5 \% \mathrm{CO}_{2}$, whereas $293 \mathrm{~T}$ cells were cultured in DMEM (Gibco, Grand Island, NY, US) supplemented with $10 \%$ FBS (HyClone, Logan, UT, USA) at $37^{\circ} \mathrm{C}$ in a humidified atmosphere containing $5 \% \mathrm{CO}_{2}$.

Plasmid vector construction and recombinant protein purification

The plasmid pGEX-4T-1-ABCE1 was a generous gift from Dr. J.R. Lingappa [5], and pGEX-4T-1$\triangle$ ABCE1 (without the Fe-S domain coding sequence) plasmids were constructed via PCR amplification using cDNA from human LUAD cells (LETP-a-2) as a template. The coding sequences were inserted into pGEX-4T-1 vectors using BamHI and EcoRI restriction endonucleases. The ABCE1 and $\triangle \mathrm{ABCE} 1$ primers were as follows: ABCE1, Forward 5'-AGTCGCGGCCGCCTAATCATCCAAGAAAAAGTAGTTTCC-3', Reverse 5'-AGTCGGATCCATGGCAGACAAGTTAACGAGAATT-3'; and $\triangle$ ABCE1, Forward 5'-GATGCGGCCGCTCGAGTCGACCCGGGAATTCTTACTAATCATCCAAGAAAAAGT-3', Reverse 5'-GATGCGGCCGCTCGAGTCGACCCGGGAATTCTTACTAATCATCCAAGAAAAAGT-3'.

After the plasmids were sequenced, pGEX-4T-1-ABCE1, pGEX-4T-1- $\triangle A B C E 1$ and the empty plasmids were transformed into E. coli BL21 (DE3) plysS cells (Tiangen, China) and grown at $37^{\circ} \mathrm{C}$ in LB broth containing $60 \mu \mathrm{g} / \mathrm{mL}$ ampicillin to an optical density of $0.5-0.6$. Induction was conducted at $25^{\circ} \mathrm{C}$ overnight using a final concentration of $0.4 \mathrm{mM}$ isopropyl-1-thio-beta-D-galactopyranoside (IPTG, Tiangen, Beijing, China). The cells were harvested via centrifugation at $5000 \times \mathrm{g}$ for 5 minutes, sonicated and subsequently collected via centrifugation at 140, 00×g (rotor JA-25.5, Beckman Coulter) for 10 minutes. Purified recombinant glutathione S-transferase-labeled ABCE1 (GST-ABCE1), glutathione S-transferase-labeled $\triangle A B C E 1$ (GST$\triangle A B C E 1$ ) and GST proteins were extracted using a recombinant protein purification kit (Millipore, Billerica, MA, USA). ATPase activity was assessed using ATPase Activity Assay Kits (Abnova, Taipei, Taiwan, China) according to the manufacturer's instructions and was calculated as follows: Enzyme activity $=[\mathrm{Pi}](\mu \mathrm{M}) \times 40$ $\mu \mathrm{L} \div(10 \mu \mathrm{L} \times t)(\mathrm{U} / \mathrm{L})$.

\section{GST pull-down assay}

A GST pull-down assay was employed to confirm the interactions between the ABCE1 Fe-S clusters and $\beta$-actin. For the GST pull-down assays, $50 \mu \mathrm{L}$ of glutathione-sepharose resin from a recombinant protein purification kit (Millipore, Billerica, MA, USA) was bound to recombinant GST-ABCE1, GST- $\triangle$ ABCE1 or GST alone without eluting and mixed with $0.4 \mathrm{mg} / \mathrm{mL}$ purified $\beta$-actin (Cytoskeleton, Denver, CO, USA) on a rotating incubator at $37^{\circ} \mathrm{C}$ for 2 hours. Then, $0.4 \mathrm{mg} / \mathrm{mL}$ purified $\beta$-actin and $0.2 \mathrm{mM}$ ATP (Cytoskeleton, Denver, CO, USA) were dissolved in reaction buffer ( $20 \mathrm{mM}$ Tris, $100 \mathrm{mM} \mathrm{NaCl}, 1 \mathrm{mM}$ DTT and $1 \mathrm{mM}$ EDTA). The supernatant was removed, and the resin was washed in washing buffer provided by the kit. Then, samples were harvested via centrifugation at $500 \times \mathrm{g}$ at $4^{\circ} \mathrm{C}$ for 5 minutes, which was repeated three times. Protein-bound resin was heat-denatured and separated using sodium dodecyl sulfate-polyacrylamide gel electrophoresis (SDS-PAGE) through a $10 \%$ gel. The gel was stained with a Coomassie Brilliant Blue Fast Staining Solution (Tiangen, Beijing, China) and scanned, after which the GST-ABCE1, GST- $\triangle$ ABCE1 and $\beta$-actin proteins were identified. Control pull-downs were similarly conducted using GST alone and glutathione-sepharose resin. Each experimental condition was assessed at least three times.

\section{Lentiviral packaging vector construction and infection}

The Lenti-Easy Packaging System (Shanghai Genechem Co., Ltd., China) was used due to its high infection and transduction efficiencies in human cells [21].

ABCE1 and $\triangle \mathrm{ABCE} 1$ were synthesized and annealed using the following primers:

ABCE1: Forward 5'-GAGGATCCCCGGGTACCGGTCGCCACCATGGCAGACAAGTTAACGAG-3', Reverse 5'-TCCTTGTAGTCCATACCATCATCCAAGAAAAAGTAGTTTC-3'; and

$\triangle$ ABCE1: Forward, 5'-GAGGATCCCCGGGTACCGGTCGCCACCATGCCAAGCAACTTGGAAAAAG-3', Reverse 5'-TCCTTGTAGTCCATACCATCATCCAAGAAAAAGT AGTTTC-3'. The GV358-ABCE1 and GV358- $\triangle$ ABCE1 lentiviral vectors were constructed. The elements within the GV358 vector and restriction enzyme used for inserting the $A B C E 1$ sequences were

Ubi-MCS-3FLAG-SV40-EGFP-IRES-puromycin and AgeI, respectively. Using the Lenti-Easy Packing Mix (Shanghai Genechem Co., Ltd., China) and Lipofectamine 2000 (Invitrogen, Carlsbad, CA, USA), the GV358ABCE1, GV358- $\triangle A B C E 1$ and control vectors were transfected into 293T cells, which were cultured in OptiMEM for 2 hours before transfection. Cells were maintained at $37^{\circ} \mathrm{C}$ in a humidified atmosphere containing 


\section{Cellular Physiology Cell Physiol Biochem 2017;44:554-566 \begin{tabular}{l|l|l} 
and Biochemistry 10.1159/000485090 & $\begin{array}{l}\text { C) } 2017 \text { The Author(s). Published by S. Karger AG, Basel } \\
\text { www.karger.com/cpb }\end{array}$ \\
\hline
\end{tabular}}

Yu/Han/Tian: Fe-S Cluster of ABCE1 and Lung Adenocarcinoma Metastasis

$5 \% \mathrm{CO}_{2}$. After 8 hours of transfection, the culture media were exchanged with fresh MEM culture medium supplemented with $10 \%$ FBS. At 72 hours after transfection, the supernatants containing lentiviral particles were harvested and collected by $1500 \mathrm{rpm}$ centrifugation for 5 minutes and filtered through a $0.22 \mu \mathrm{m}$ PES filter (Millipore, Billerica, MA, USA). The lentiviral particles were concentrated in ultrafiltration centrifugal tubes (Centricon Plus-20, Millipore, Billerica, MA, USA). To quantify each vector titer, 293T cells were infected with various dilutions of lentiviral particles, and the number of cells expressing green fluorescent protein were counted at 96 hours after infection. The viral titer was calculated as follows: Quantity of cells $/ 10^{-5}$ (volume of vector original liquid $(\mu \mathrm{L})$ ) $=$ Titer $(\mathrm{TU} / \mathrm{ml})$.

Subsequently, lentiviral packaging vectors with multiplicities of infection (MOIs) of 15 were mixed with F12K culture medium containing $5 \mu \mathrm{g} / \mathrm{mL}$ polybrene (Shanghai Genechem Co., Ltd., China). The lentiviral packaging vector solution was added at a 30\% infection rate per well to A549 cells in 6-well plates for 24 hours. Subsequently, the medium was exchanged for fresh complete F12K culture medium for an additional 48 hours. Finally, fluorescence microscopy was used to evaluate the infection rate, which was calculated as follows: (Quantity of cells expressed green fluorescent protein in dark field / Quantity of cells in bright field) $\times 100 \%=$ Rate of infection.

Western blot analysis

A549 cells were lysed in radioimmunoprecipitation assay (RIPA) buffer (50 mM Tris- $\mathrm{HCl}$ [pH 7.4], $150 \mathrm{mM} \mathrm{NaCl}, 1 \% \mathrm{NP}-40,0.1 \%$ sodium dodecyl sulfate, $0.5 \%$ sodium deoxycholate). After the proteins were pelleting, a BCA protein assay was conducted, after which the proteins were denatured and separated using SDS-PAGE on a $10 \%$ gel. The separated proteins were then transferred to polyvinylidene difluoride (PVDF) membranes (Millipore, Bedford, MA, USA), which were incubated with either anti-ABCE1 antibody [ERP15373(B)]-C-terminal (1:5000, Abcam, Cambridge, MA, USA) or a GAPDH monoclonal antibody (1:1000, Bioss, Beijing, China) and subsequently incubated with a peroxidase-conjugated AffiniPure goat anti-rabbit IgG $(\mathrm{H}+\mathrm{L})$ antibody (1:2000, ZSGB-Bio, Beijing, China). Signals on the membranes were detected using enhanced chemiluminescence (ECL, Tanon, Shanghai, China). Each experiment was independently repeated three times. Densitometry evaluation of ABCE1 and GAPDH expression was analyzed using ImageJ $1.47 \mathrm{v}$ software, and ratios of densitometry value of ABCE1/GAPDH were calculated and compared.

\section{Transwell assay}

The migration of A549 cells was assessed using Transwell inserts (Corning, Corning, NY, US). At 48 hours after transfection, $2 \times 10^{4}$ cells in $200 \mu \mathrm{L}$ of serum-free $\mathrm{F} 12 \mathrm{~K}$ medium were seeded into the upper chamber (which was placed in the well of a 24 -well plate), and $600 \mu \mathrm{L}$ of F12K medium supplemented with $10 \%$ FBS was added to each well of the plate as a chemo-attractant to encourage cell migration. After the cells incubated for 48 hours, those that did not migrate were removed using a cotton swab. All remaining cells were stained using $1 \%$ crystal violet and counted under a light microscope. The number of migratory cells was counted in 4 fields under $200 \times$ magnification to determine the average number of migratory cells. Each experiment was independently repeated three times [22].

\section{Wound healing assay}

Cells in the logarithmic growth phase were plated into a 24 -well plate and cultured at $37^{\circ} \mathrm{C}$ in a humidified atmosphere containing $5 \% \mathrm{CO}_{2}$. The infection rate was greater than $90 \%$ after 24 hours, after which a scratch was made on the cell monolayer using a sterile 200- $\mu$ L pipette tip (Axygen, Union, CA, USA). Images of the scratch under a light microscope (Nikon Eclipse TE2000-S, Japan) were collected at 0, 24 and 48 hours after scratching, and the space width was analyzed using Image-Pro plus Version 6.0.0.260 software. The wound healing assay was performed in triplicate.

\section{MTT (3-(45,)-dimethylthiahiazo (-z-y1)-35, -di- phenytetrazoliumromide) test}

An MTT assay was performed to measure the viability of the cultures. Cells were treated with the different lentiviral constructs described above for 48 hours. Then, $1 \times 10^{3}$ cells in $200 \mu \mathrm{L}$ were plated in 96well plates. After culturing for 2, 24, 48, 72 or 96 hours, $180 \mu \mathrm{L}$ of $10 \%$ F12K medium and $20 \mu \mathrm{L}$ of MTT solution ( $5 \mathrm{mg} / \mathrm{mL}$ in phosphate-buffered saline (PBS)) were added to each well, and the treated cells were incubated at $37^{\circ} \mathrm{C}$ for 4 hours. Then, the supernatants were removed, and $150 \mu \mathrm{L}$ dimethyl sulfoxide (DMSO) was added into each well. The plates were agitated at room temperature for 10 minutes to dissolve the 


\section{Cellular Physiology Cell Physiol Biochem 2017;44:554-566

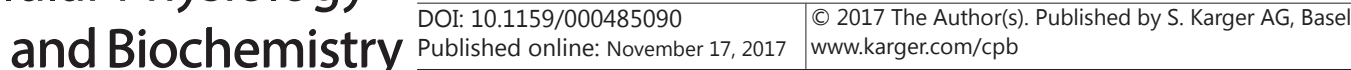 \\ Yu/Han/Tian: Fe-S Cluster of ABCE1 and Lung Adenocarcinoma Metastasis}

dark-blue MTT crystals. The optical densities of the cultures were read on an Autobio Microplate Reader (Autobio Diagnostics Co., Zhengzhou, China) at $570 \mathrm{~nm}$. The MTT assay was performed in three independent experiments.

\section{Clonogenic assay}

A549 cells were trypsinized, resuspended in medium and counted. Cells (300 per well) were reseeded in 6-well plates and incubated for 12 days at $37{ }^{\circ} \mathrm{C}$ in a humidified atmosphere containing $5 \% \mathrm{CO}_{2}$. After the media were aspirated, the cells were washed twice with ice-cold PBS. The cell colonies were sequentially fixed in 4\% paraformaldehyde for 30 minutes and 95\% ethanol for 10 minutes, after which they were stained with $1 \%$ crystal violet for 30 minutes on a decolorization table and counted. Three independent experiments were performed.

\section{Cytoskeleton staining}

A549 cells infected with the different lentiviral vectors were separately seeded in confocal plates and cultured for 24 hours. The culture media were removed, and the cells were washed once with PBS and fixed in $4 \%$ paraformaldehyde for 10 minutes at room temperature. After the cells were washed again with PBS at room temperature for 30 seconds, they were permeabilized in permeabilization buffer $(0.5 \%$ Triton X-100 in PBS) at room temperature for 5 minutes and washed with PBS for 30 seconds. Then, $200 \mu \mathrm{L}$ of $100 \mathrm{nM}$ rhodamine phalloidin (Cytoskeleton, Denver, CO, USA) was added to the cells. The samples were incubated in a dark, humid chamber at room temperature for 30 minutes followed by three washes with PBS. Cell nuclei were visualized by counterstaining the cells with $200 \mu \mathrm{L}$ of $100 \mathrm{nM}$ 4,6-diamidino-2-phenylindole (DAPI) in PBS for 10 minutes. The stained cells were washed three times with PBS. Finally, fluorescent images of the stained cells were captured using a confocal laser scanning microscope (Olympus, Japan) and visualized using Olympus FA10-ASW 3.0 Viewer software.

\section{Statistical analysis}

All statistical analyses were performed using SPSS 19.0 software. Quantitative values were presented as the means \pm SD. A one-way ANOVA followed by a Tukey-Kramer multiple comparisons test was conducted to compare corresponding data. The results were considered significant at $P<0.05$.

\section{Results}

Induction, purification and ATPase activity of recombinant GST-ABCE1 and GST- $\triangle A B C E 1$

The pGEX-4T-1-ABCE1 and pGEX-4T-1- $\triangle \mathrm{ABCE1}$ plasmids, which contained full-length ABCE1 and the Fe-S cluster-deficient coding sequence (210 bp at the N-terminal tail) $\triangle \mathrm{ABCE} 1$, respectively, were constructed. The sequences inserted into the recombinant plasmids were verified, and their efficacies were tested in vitro. Coomassie Blue staining indicated a band of purified GST-ABCE above $95 \mathrm{kDa}$ in lane 6, a band of purified GST- $\triangle \mathrm{ABCE} 1$ between 72 $\mathrm{kDa}$ to $95 \mathrm{kDa}$ in lane 8 and band of GST between $26 \mathrm{kDa}$ to $34 \mathrm{kDa}$ in lane 4 (corresponding to the pGEX-4T-1 plasmid) after the plasmids were transformed into BL21 (DE3) cells and the bacteria were induced. These results show the successful induction of GST-ABCE1, GST$\triangle \mathrm{ABCE} 1$ and GST by IPTG (Fig. 1A). We subsequently purified the recombinant GST proteins to $1 \mathrm{pmol} / \mu \mathrm{L}$ and evaluated their biological activity. As shown in Fig. $1 \mathrm{~B}$, the recombinant ABCE1 and $\triangle \mathrm{ABCE} 1$ proteins had higher ATPase activity for ATP hydrolysis than GST protein alone $(P<0.05)$. Furthermore, the activity of GST-ABCE1 was higher than that of GST- $\triangle \mathrm{ABCE} 1$ $(P<0.05)$.

The Fe-S cluster is essential for specific interactions between ABCE1 and $\beta$-actin

The GST pull-down assay was used to verify a possible interaction between ABCE1 and $\beta$-actin. Purified $\beta$-actin protein was individually mixed with a glutathione-sepharose resin bound to recombinant GST-ABCE1, GST- $\triangle$ ABCE1 or GST protein. As shown in Fig. 2A, two bands above $95 \mathrm{kDa}$ and $43 \mathrm{kDa}$ representing GST-ABCE1 and $\beta$-actin, respectively, were detected by Coomassie Blue stain in lane 2. $\beta$-Actin binding to either GST alone or the 
Fig. 1. Induction, purification and biological assessment of recombinant $\mathrm{ABCE} 1$ and $\triangle \mathrm{ABCE} 1$. A) $\mathrm{Pu}$ rification of recombinant GST-ABCE1, GST$\triangle \mathrm{ABCE1}$ and GST was detected using SDSPAGE and Coomassie Brilliant Blue staining. Lanes 1, 5, 7, Marker; lane 2, elution of purified GST-ABCE1; lane

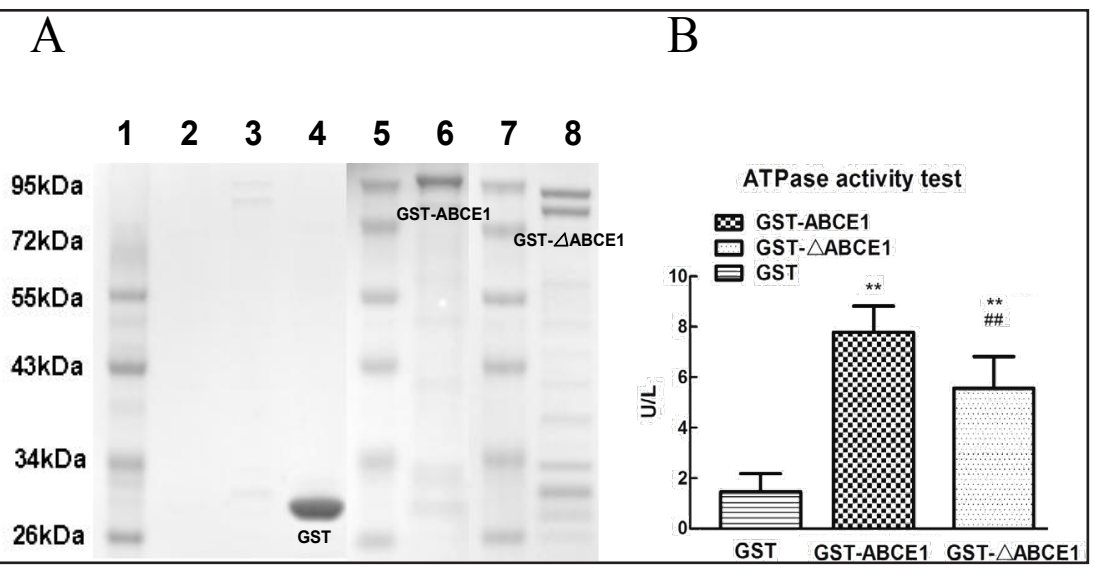

3 , elution of purified

GST- $\triangle \mathrm{ABCE} 1$; nonobvious bands were observed in lanes 2 and 3 due to low protein concentrations caused by low protein solubility; lane 4, purified GST, between $26 \mathrm{kDa}$ and $34 \mathrm{kDa}$; lane 6, purified GST-ABCE1, over $95 \mathrm{kDa}$; lane 8, purified GST- $\triangle \mathrm{ABCE} 1$, between $72 \mathrm{kDa}$ and $95 \mathrm{kDa}$. B) ATPase activity of the purified GSTABCE1, GST- $\triangle \mathrm{ABCE} 1$ and GST proteins. Data are expressed as the mean \pm SEM. ${ }^{* *} P<0.05$ vs. GST protein; \#\# $P<0.05$ vs. GST-ABCE1.

Fig. 2. GST pull-down assay demonstrated the essential role of Fe-S clusters in the in vitro interaction between ABCE1 and $\beta$-actin. A) Interaction between GST-ABCE1 and purified $\beta$-actin. Lane 1, Marker. Lane 2, GST-ABCE1 bound to glutathione-sepharose resin beads (GS) were mixed with $\beta$-actin and ATP. There were two bands as indicated by Coomassie Brilliant Blue staining. The band at $95 \mathrm{kDa}$ represents GST-ABCE1 and the other band at $43 \mathrm{kDa}$ represents $\beta$-actin, implying a specific binding interaction between GSTABCE1 and $\beta$-actin. Lane 3, GST-ABCE1 bound to glutathione-sepharose resin beads (GS) as a control. Lane 4, GST protein bound to glutathione-sepharose resin beads (GS) was mixed with purified $\beta$-actin. Only one band below $34 \mathrm{kDa}$ was present, suggesting that GST alone could not bind with $\beta$-actin. Lane 5 , GST protein combined with glutathione-sepharose resin beads (GS) as a con-

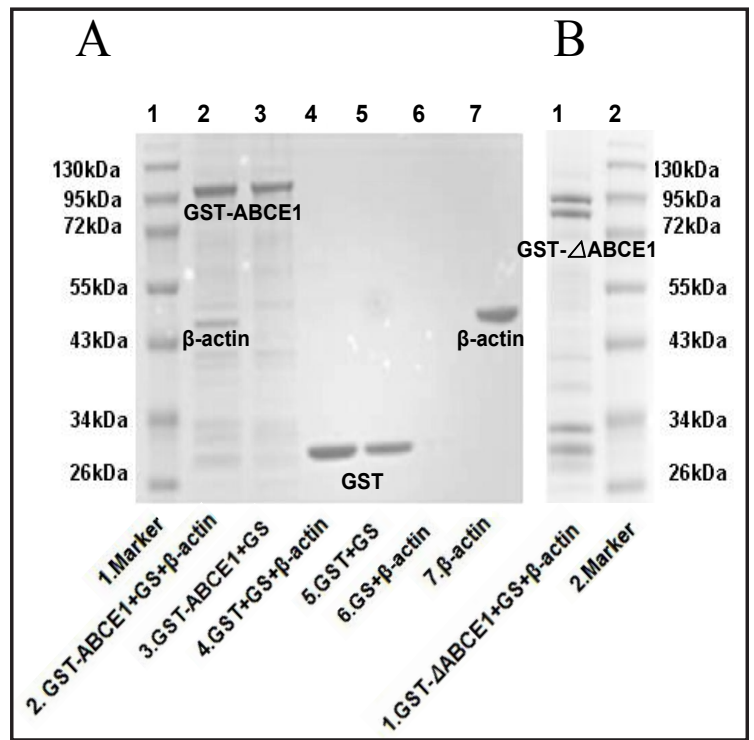
trol. Lane 6, glutathione-sepharose resin beads (GS) combined with $\beta$-actin. There was no band present. Lane 7 , the band at $43 \mathrm{kDa}$ represents $\beta$-actin. B) Interaction of GST- $\triangle$ ABCE1 and purified $\beta$-actin. In lane 1 , there were only bands present between $72 \mathrm{kDa} \sim 95 \mathrm{kDa}$ (approximately $85 \mathrm{kDa}$ ), which represented GST- $\triangle \mathrm{ABCE} 1$ and indicated $\beta$-actin was absent. This result demonstrates that GST- $\triangle \mathrm{ABCE} 1$ was not able to interact with $\beta$-actin due to the loss of the Fe-S cluster domain.

glutathione-sepharose resin beads was not observed in lanes 4 or 6 , but $\beta$-actin was detected in the control (lane 7). These results suggest a specific interaction between GST-ABCE1 and $\beta$-actin. Similarly, lane 2 in Fig. 2B shows that bands were only present at approximate $85 \mathrm{kDa}$ (between $95 \mathrm{kDa}$ and $72 \mathrm{kDa}$ ), which corresponds to GST- $\triangle \mathrm{ABCE} 1$, and there was no band at approximately $43 \mathrm{kDa}$, which corresponds to $\beta$-actin. This result indicating the crucial role of the Fe-S cluster domains of ABCE1 in activities involving $\beta$-actin. 
Fig. 3. Infection efficiency of various lentiviral packaging vectors and corresponding target protein expression. A) Detection of infection efficiency in A549 cells using fluorescence microscopy. Observation of the green fluorescence in A549 cells indicated an infection efficiency greater than $95 \%$ at 72 hours after infection. B) Western blot

measurement of ABCE1 and $\triangle \mathrm{ABCE} 1$ expression in A549 cells transduced with lentiviral vectors. Proteins were extracted from control cells and A549 cells infected with lentivirus that either overexpressed ABCE1 or $\triangle \mathrm{ABCE} 1$ or contained an empty vector. Lane 1, samples of A549 cells infected with lentivirus overexpressing $\triangle \mathrm{ABCE} 1$. Two bands were approximately located at the position of destination protein; the superior one is native $A B C E 1$, and the inferior one is $\triangle A B C E 1$. Lane 2, samples of A549 cells infected with lentivirus overexpressing ABCE1. Lane 3, samples of A549 cells infected with lentivirus containing empty vector. Lane 4 , samples of untreated control cells. Data are expressed as the mean \pm SEM. ${ }^{* *} P<0.05$.

Fig. 4. The

Fe-S cluster domain is essential for tumor g r o w t h potential. A) Clonogenic assays were performed to observe the proliferation of cells infected with lentiviral packaging

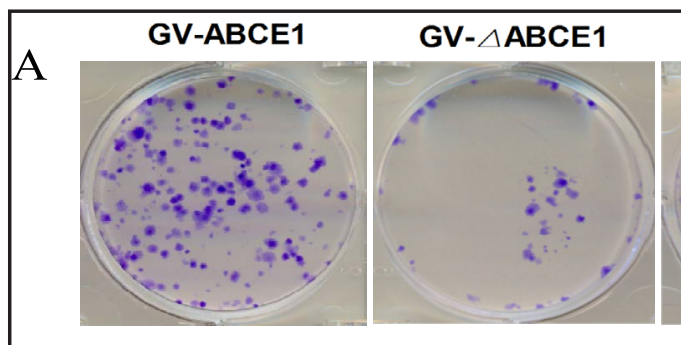

B

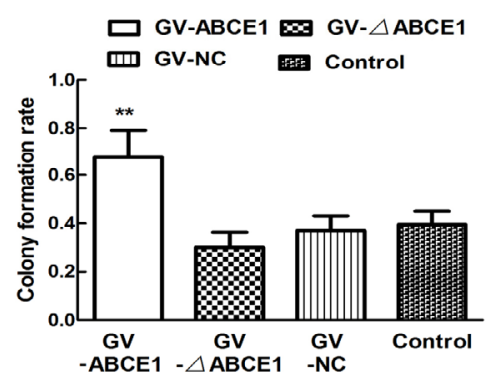

GV- NC

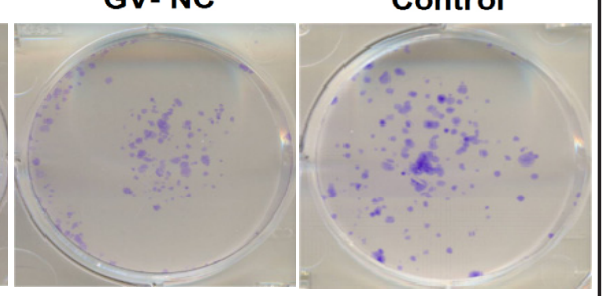

C

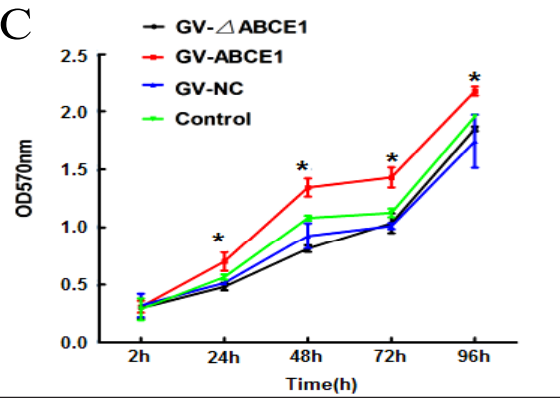

ve c tors.

Representative images are shown. B) Quantification of colonies formed by control cells and cells infected with lentiviral packaging vectors indicating that the growth potential enhanced by ABCE1 was significantly decreased when in cells lacking a functional Fe-S cluster $\left({ }^{*} \mathrm{P}<0.05\right)$. C) The effects of the Fe-S cluster in ABCE1 on the proliferative potential of infected cells were determined using the MTT assay. ${ }^{* *} \mathrm{P}<0.05$.

Construction and identification of lentiviral packaging vectors for overexpression of $A B C E 1$ and $\triangle A B C E 1$

To determine whether the Fe-S cluster domains of ABCE1 played essential roles in the migration and proliferation of tumor cells, we ectopically expressed ABCE1 and $\triangle A B C E 1$ by 


\section{Cellular Physiology Cell Physiol Biochem 2017;44:554-566 \begin{tabular}{ll|l} 
DOI: 10.1159/000485090 & $\begin{array}{l}\text { O 2017 The Author(s). Published by S. Karger AG, Basel } \\
\text { www.karger.com/cpb }\end{array}$
\end{tabular} \\ Yu/Han/Tian: Fe-S Cluster of ABCE1 and Lung Adenocarcinoma Metastasis}

infecting cell lines with lentivirus containing overexpression plasmids. The specific ABCE1 and $\triangle \mathrm{ABCE} 1$ coding regions were cloned into GV358-GFP plasmids and confirmed by sequencing. The resultant GV-ABCE1 and GV- $\triangle \mathrm{ABCE} 1$ plasmids as well as negative control vectors were transfected into 293T cells. After 8 hours, protein expression was detected using a fluorescence microscope (Fig. 3A). The supernatants were collected to isolate the lentiviral particles containing the GV-ABCE1, GV- $\triangle \mathrm{ABCE} 1$ and control plasmids, and these lentiviral particles were used to infect A549 cells. Protein expression was observed in infected cells under a fluorescence microscope 72 hours after infection. The infection efficiency reached $95 \%$ based on the GFP fluorescence signals (Fig. 3A). To assess the expression of ABCE1 and $\triangle A B C E 1$ in the infected A549, the proteins were extracted from the infected and control cells and analyzed using western blotting (Fig. 3B). Given the essential role of ABCE1, protein bands at $68 \mathrm{kDa}$ indicative of ABCE1 were observed in all the groups. However, the levels of ABCE1 in cells infected with GV-ABCE1 were much higher than those in the control cells or in cells infected with either GV- $\triangle A B C E 1$ or GV-NC (negative control) $(P<0.05)$. Similarly, $\triangle \mathrm{ABCE} 1$ appeared at $58 \mathrm{kDa}$ just below native ABCE1 in lane 1 and was only detected in cells infected with GV- $\triangle \mathrm{ABCE} 1$. This result indicated that native protein expression was not affected by the lentiviral vectors.

\section{Effects of the ABCE1 Fe-S cluster on A549 cell growth potential and viability}

Once we demonstrated that the Fe-S cluster domain was closely associated with $\beta$-actin, we further considered whether this unique ABCE1 domain was responsible for tumor properties associated with actin cytoskeleton remodeling. The most obvious approach was to assess cell proliferation using clonogenic assays. We observed more pronounced and more frequent colony formation in cells overexpressing ABCE1 induced by GV-ABCE1 (Fig. 4A, $P$ $<0.05$ ). However, no significant difference in the number of colonies formed was observed between cells infected with either GV- $\triangle A B C E 1$ or GV-NC and untreated A549 cells (Fig. 4B, $P>0.05)$. We also performed MTT assays to investigate the effects of ABCE1 overexpression on cell viability. As expected, the cells lentivirally infected with GV-ABCE1 proliferated much faster than the other treated cells (Fig. 4C, $P<0.05$ ). Comparatively, cells expressing $\triangle \mathrm{ABCE} 1$ (i.e., ABCE1 with a lack of Fe-S clusters) showed ineffective proliferation rates $(P>0.05)$. There were no marked differences between untreated cells and cells infected with GV-NC $(P$ $>0.05$ ).

\section{Effects of the ABCE1 Fe-S clusters on the migratory potential of A549 cells}

The metastatic potential of a tumor is dependent on the ability of tumor cells to migrate to distant sites. To assess whether the Fe-S domain affects the migration potential of A549 cells, Transwell and wound healing assays were performed in vitro. In the Transwell assay, high concentrations of chemoattractants (i.e., FBS) encouraged cell migration to the lower chamber (Fig. 5A and Fig. 5B). As shown in Fig. 5C, the migratory ability was significantly enhanced in cells infected GV-ABCE1. This effect was absent in cells infected with GV$\triangle$ ABCE1 (Fig. 5C, $P<0.05$ ). Consistent with the results of the Transwell assays, the speed of wound closure in the wound healing assays progressively increased in cells overexpressing ABCE1 (Fig. 5D, $P<0.05$ ). At 48 hours after scraping, the migratory distance of the GV-ABCE1 cells into the wound region was $1078.98 \pm 27.77$, whereas the migratory distance of cells expressing ABCE1 lacking the Fe-S cluster (GV- $\triangle \mathrm{ABCE} 1$ ) was approximately 45\% shorter (590.77 \pm 35.91 , Fig. 5D, $P<0.05$ ). There was no significant difference in the migration distance between the control cells and cells infected with GV-NC $(P>0.05)$.

The actin cytoskeleton is responsible for changes in cell shape during cell migration. We used rhodamine phalloidin, GFP and DAPI to monitor actin cytoskeleton polymerization and morphological changes in infected A549 cells. Representative images showed distinct morphological changes in cells with lentiviral-mediated upregulation in ABCE1 expression. GV-ABCE1 A549 cells presented a higher frequency of cell membrane processes associated with increased cytoskeletal polymerization and polarization as indicated by the stronger phalloidin labeling intensity (Fig. 5E, $P<0.05$ ). Cells expressing the ABCE1 construct lacking 


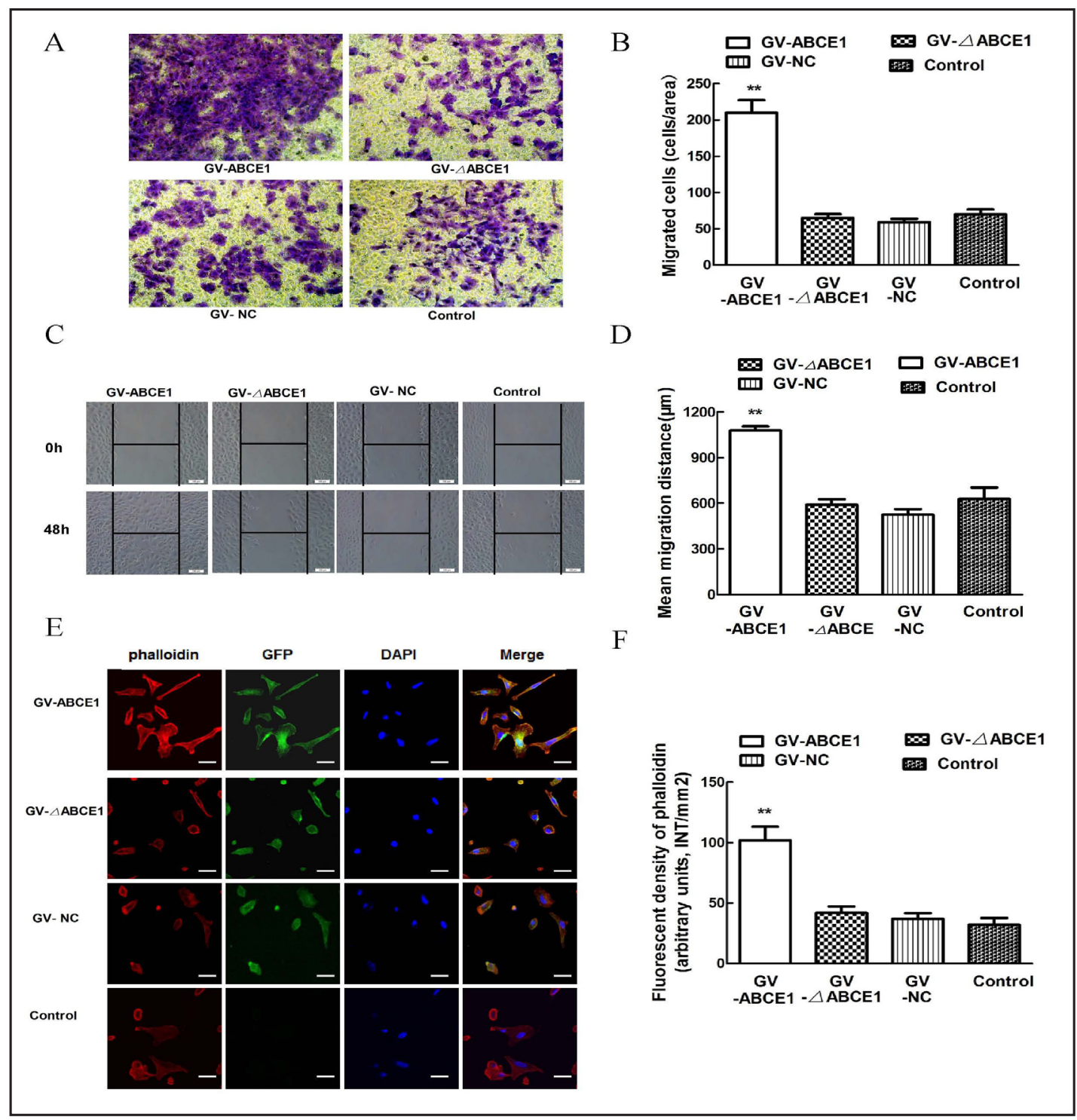

Fig. 5. Fe-S cluster domain is essential in ABCE1-mediated tumor migration potential. A) Cell migration was measured in Transwell chambers. Representative images are shown at 48 hours after experiment initiation. B) Quantification of migration after 48 hours indicating that compared to cells that overexpressed ABCE1, cells that overexpressed mutant ABCE1 lacking the Fe-S cluster exhibited a greater than $50 \%$ reduction in the migratory ability. C) Cell mobility was measured using the wound healing assay. Scratches were made in confluent monolayers with a pipette tip. Representative wound healing images at 0 and 48 hours. D) Quantification of wound healing indicating that the migration distance across the wounding region was obviously increased in cells overexpressed ABCE1, which was abrogated in cells infected with GV- $\triangle \mathrm{ABCE} 1$. Data are expressed as the mean $\pm \operatorname{SEM}^{* *} P<0.05$. E) Representative images of immunofluorescence staining of the cytoskeleton-related protein phalloidin in A549 cells infected with lentivirus. Co-localization (yellow) of phalloidin (red) and lentiviral GV358-GFP (green) as well as nuclear staining (blue) is demonstrated on the merged images. Cells overexpressing ABCE1 at 48 hours after infection presented a higher presence of cell processes at the cell membrane, which were absent in cells infected with GV- $\triangle$ ABCE1. Data are expressed as the mean \pm SEM. ${ }^{* *} P<0.05$.

the Fe-S clusters showed smoother membranes and minimal cytoskeletal rearrangement. The fluorescent density of phalloidin was $102.00 \pm 11.24$ and $42.00 \pm 5.33$ in cells infected with GV-ABCE1 and GV- $\triangle$ ABCE1, respectively (Fig. 5F, $P<0.05$ ). These data suggest that the 


\section{Cellular Physiology Cell Physiol Biochem 2017;44:554-566 \begin{tabular}{l|l|l} 
DOI: 10.1159/000485090 & $\begin{array}{l}\text { O 2017 The Author(s). Published by S. Karger AG, Basel } \\
\text { www.karger.com/cpb }\end{array}$
\end{tabular}}

Yu/Han/Tian: Fe-S Cluster of ABCE1 and Lung Adenocarcinoma Metastasis

ABCE1 Fe-S clusters have a significant effect on the migratory activity of A549 cells that is likely due to cytoskeletal polymerization.

\section{Discussion}

Lung cancer is the leading cause of mortality of all malignant tumors worldwide [1]. The present study revealed for the first time the functional relevance of the ABCE1 Fe-S clusters in the migration and metastasis of human LUAD and in actin cytoskeletal rearrangement via interactions with $\beta$-actin.

ABCE1 is a highly conserved protein that contains unique Fe-S clusters in the $\mathrm{N}$-terminal region. $A B C E 1$ codes for ribonuclease $L$ inhibitor, which regulates various biological functions, such as ribosome biosynthesis and recycling, as well as HIV capsid assembly [23, 24].

Although an increasing number of studies has demonstrated the role of ABCE1 in tumor cell development, very little is known about the actual mechanisms [7, 25]. Furthermore, the roles of ABCE1 and its Fe-S clusters in carcinogenesis are still undetermined. Previous studies have reported that the mRNA and protein expression levels of $\beta$-actin were significantly higher in tumor tissues than in normal adjacent tissues [26, 27]. Other studies further identified $\beta$-actin reorganization as important in tumor cell migration [18]. Reports on metastatic cancer cells indicated that $\beta$-actin was transformed to a dispersed state, i.e., diffusely localized throughout the cytoplasm and distributed in regions of lamellar activity instead of at intercellular junctions [28]. To explore the potential interactions between $\mathrm{ABCE} 1$ and $\beta$-actin, we hypothesized that the Fe-S cluster, one most important functional domain of ABCE1, may mediate $\beta$-actin cytoskeletal rearrangements. Thus, we first obtained purified recombinant proteins by transforming cells with plasmids containing either fulllength or Fe-S cluster-deficient coding sequences (ABCE1 and $\triangle A B C E 1$, respectively). ATP hydrolysis is an important function of ABCE1, so we tested ATP hydrolysis activity to evaluate biological activity of the recombinant proteins. Proteins exhibiting native biological activity could be used to conduct further experiments. Both GST-ABCE1 and GST- $\triangle$ ABCE1 exhibited more ATPase activity than the GST protein alone, and the ATPase activity of GST- $\triangle$ ABCE1 was lower than that of GST-ABCE1. However, previous studies on ABCE1 from Sulfolobus solfataricus reported by Barthelme et al. suggest that neither the prosthetic group nor the lack of the entire Fe-S cluster domain affected ATPase activity at $80^{\circ} \mathrm{C}$ [11]. Other studies on ABCE from Pyrococcus abyssi by Sims and Igarashi suggest that the ATPase activity is jointly regulated by the status of $\mathrm{Fe}-\mathrm{S}$ and $\mathrm{Mg}^{2+}$, as PabABCE1 without its full complement of Fe-S clusters is more sensitive to $\mathrm{Mg}^{2+}$ inhibition and overall energy charge under strict anaerobic conditions at $60-85^{\circ} \mathrm{C}$ [29]. In our studies, ATPase activity was assessed under aerobic conditions at room temperature without precise $\mathrm{Mg}^{2+}$ titration. The conformation and oxidation state of the GST recombinant protein, temperature, $\mathrm{Mg}^{2+}$ concentration and presence of the Fe-S cluster domain may influence enzyme activity.

The GST pull-down assay is commonly used to verify interactions between proteins. As shown in Fig. 2, the two bands stained with Coomassie Blue were detected only when $\beta$-actin was bound to GST-ABCE1. Under other conditions, bands of either recombinant protein or $\beta$-actin were detected in each lane (lanes 3-7 in Fig. 2A, lane 1 in Fig. 2B), demonstrating the crucial role of the Fe-S cluster domain in ABCE1 binding to $\beta$-actin. The expected band of GST$\triangle \mathrm{ABCE} 1$ is approximately $85 \mathrm{kDa}$; however, there is an additional band at a lower position. Studies on the structure of the ribosome post-recycling complex by Kiosze-Becker K et al. suggested the Fe-S cluster domain anchors to NBD1 via a two $\beta$-strand lever arm; this lever arm swings to rotate the Fe-S cluster domain 160 degrees. In addition, there is a helix-loophelix (HLH) at the beginning of NBD1 [12]. We speculate that when the Fe-S cluster domain was knocked out, the unstable lever arm and HLH regions underwent either abscission or hydrolysis from NBD1 resulting an incomplete recombinant protein. The molecular weight of the incomplete GST- $\triangle \mathrm{ABCE} 1$ is slightly lower than that of the complete protein.

In gene function studies, the specific knockdown or upregulation of target genes without affecting other gene products is an important method for developing specific mutants for 
use in lentiviral packaging vectors [30-32]. In view of the vital function of ABCE1 in protein synthesis, translation termination and ribosome recycling, knockdown of ABCE1 may interfere with basic cellular metabolism. In this study, we constructed lentiviral packaging vectors to overexpress $A B C E 1$ and $\triangle A B C E 1$ in A549 cells to avoid the potential side effects from knocking down or knocking out ABCE1. Using fluorescence microscopy and western blotting, we observed that the target proteins were overexpressed as shown in Fig. 3. Rapid growth and increased metastatic potential are two basic characteristics of tumor cells. Recent evidence has indicated that ABCE1 is crucial in tumor progression, prognosis and metastasis [7, 9]. Our MTT and clonogenic assay data showed that A549 cells overexpressing ABCE1 grew much faster and formed more pronounced and a larger number of colonies. Cells expressing mutant ABCE lacking the Fe-S clusters showed opposing effects. Cell proliferation was obviously inhibited when ABCE1 was down-regulated [7, 8]. Collectively, the data suggest that the Fe-S cluster domains have a remarkable influence on the cellular growth potential of A549 cells. We speculated that $\triangle \mathrm{ABCE} 1$ couldn't attach to or detach from the small ribosomal subunit such that the absence of the Fe-S cluster domain influenced the activity of ABCE1 regarding ribosomal protein recycling. Acceleration of proliferation caused by ABCE1 overexpression was blocked in cells expressing the Fe-S cluster domain deletion mutant. Additionally, we found that the Fe-S cluster domains could affect cell shape and migratory behavior. Previous studies have indicated that cell migration is dependent on lamellipodia formation $[32,33]$. Changes in cell polarity and actin organization may reflect alterations in cell migration. Using Transwell and wound healing assays, we observed that the migratory abilities were significantly increased in A549 cells overexpressing ABCE1 unlike the cells expressing mutant ABCE that lacked the Fe-S clusters.

To further analyze cytoskeletal organization, we used phalloidin, DAPI, and GFP to observe morphological changes in transduced A549 cells. Fluorescent signals from the phalloidin (red), lentiviral GV358-GFP (green) and nuclei (blue) images were merged to create images of the cytoskeletal organization. As expected, cells transfected with GV-ABCE1 were polygonal in shape with increased cytoskeleton polymerization as indicated by strong phalloidin staining. Cells expressing the ABCE mutant lacking the Fe-S clusters had more rounded morphology with weakly rearranged cytoskeletons. Furthermore, the average fluorescent densities of these cells verified the morphological observations in accordance with previous studies by Visegrady et al. [34]. Other authors found that reduced integrinmediated adhesion was attributed to the disturbance of F-actin (formed by $\beta$-actin) and was responsible for prostate cancer stemness and bone metastasis [32].

Unfortunately, we were unable to determine a definitive role of the Fe-S clusters. The in vitro conditions do not accurately reproduce the complex intracellular environment and cross-talk between cells. Further in vivo experiments focusing on the effects of the Fe-S domain are necessary.

Our study was the first to demonstrate that specific in vitro binding of ABCE1 to $\beta$-actin required the Fe-S cluster domain. These results highlighted the indispensable roles of the Fe-S clusters in the growth and metastatic potential of LUAD cells. The Fe-S clusters on ABCE1 may be a potential target in the prevention of lung cancer metastasis.

\section{Acknowledgements}

Funding for this project was provided by the National Natural Science Foundation of China (No. 30973502).

\section{Disclosure Statement}

The authors have no Disclosure Statement to declare. 


\section{Cellular Physiology Cell Physiol Biochem 2017;44:554-566 \begin{tabular}{l|l|l} 
and Biochemistry Published online: November 17, 2017 & $\begin{array}{l}\text { (c) } 2017 \text { The Author(s). Published by S. Karger AG, Basel } \\
\text { www.karger.com/cpb }\end{array}$ \\
\hline
\end{tabular}}

Yu/Han/Tian: Fe-S Cluster of ABCE1 and Lung Adenocarcinoma Metastasis

\section{References}

1 Ferlay J, Soerjomataram I, Dikshit R, Eser S, Mathers C, Rebelo M, Parkin DM, Forman D, Bray F: Cancer incidence and mortality worldwide: Sources, methods and major patterns in GLOBOCAN 2012. Int J Cancer 2015;136:E359-E386.

2 Zagryazhskaya A, Gyuraszova K, Zhivotovsky B: Cell death in cancer therapy of lung adenocarcinoma. Int J Dev Biol 2015;59:119-129.

3 Bisbal C, Martinand C, Silhol M, Lebleu B, Salehzada T: Cloning and characterization of a RNase L inhibitor. A new component of the interferon-regulated 2-5A pathway. J Biol chemChem 1995;270:13308-13317.

4 Catherine B, Camille M, Michelle S, Bernard L, Tamim S: Cloning and characterizaion of a RNase L inhibitor a new component of interferon-regulated 2-5A pathway. J Biol 1995;270:13308-13317.

5 Lingappa JR, Dooher JE, Newman MA, Kiser PK, Klein KC: Basic residues in the nucleocapsid domain of Gag are required for interaction of HIV-1 gag with ABCE1 (HP68), a cellular protein important for HIV-1 capsid assembly. J Biol Chem 2006;281:3773-3784.

6 Bhattacharya A, McIntosh KB, Willis IM, Warner JR: Why Dom34 stimulates growth of cells with defects of 40S ribosomal subunit biosynthesis. Mol Cell Biol 2010;30:5562-5571.

7 Tian Y, Tian X, Han X, Chen Y, Song CY, Jiang WJ, Tian DL:ABCE1 plays an essential role in lung cancer progression and metastasis. Tumour Biol 2016;37:8375-8382.

8 Huang B, Zhou H, Lang X, Liu Z: siRNA-induced ABCE1 silencing inhibits proliferation and invasion of breast cancer cells. Mol Med Rep 2014;10:1685-1690.

-9 Ren Y, Li Y, Tian D: Role of the ABCE1 gene in human lung adenocarcinoma. Oncol Rep 2012;27:965-970.

10 Barthelme D, Scheele U, Dinkelaker S, Janoschka A, Macmillan F, Albers SV, Driessen AJ, Stagni MS, Bill E, Meyer-Klaucke W, Schünemann V, Tampé R:Structural organization of essential iron-sulfur clusters in the evolutionarily highly conserved ATP-binding cassette protein ABCE1. J Biol Chem 2007;282:14598-14607.

11 Barthelme D, Dinkelaker S,Albers SV, Londei P, Ermler U, Tampé R: Ribosome recycling depends on a mechanistic link between the FeS cluster domain and a conformational switch of the twin-ATPase ABCE1. Proc Natl Acad Sci U S A 2011;108:3228-3233.

-12 Kiosze-Becker K, Ori A, Gerovac M, Heuer A, Nürenberg-Goloub E, Rashid UJ, Becker T, Beckmann R, Beck M, Tampé R: Structure of the ribosome post-recycling complex probed by chemical cross-linking and mass spectrometry. Nat Commun 2016;7:13248.

13 Sun L, Zheng J, Wang Q, Song R, Liu H, Meng R, Tao T, Si Y, Jiang W, He J: NHERF1 regulates actin cytoskeleton organization through modulation of $\alpha$-actinin-4 stability. FASEB J 2016;30:578-589.

14 Sun BO, Fang Y, Li Z, Chen Z, Xiang J: Role of cellular cytoskeleton in epithelial-mesenchymal transition process during cancer progression. Biomed Rep 2015;3:603-610.

15 Lanier MH, Kim T, Cooper JA: CARMIL2 is a novel molecular connection between vimentin and actin essential for cell migration and invadopodia formation. Mol Biol Cell 2015;26:4577-4588.

-16 Stock AM, Klee F, Edlund K, Grinberg M, Hammad S, Marchan R, Cadenas C, Niggemann B, Zänker KS, Rahnenführer J, Schmidt M, Hengstler JG, Entschladen F: Gelsolin is associated with longer metastasisfree survival and reduced cell migration in estrogen receptor-positive breast cancer. Anticancer Res 2015;35:5277-5285.

-17 Tan Q Cui J, Huang J, Ding Z, Lin H, Niu X, Li Z, Wang G, Luo Q Lu S: Genomic Alteration During Metastasis of Lung Adenocarcinoma. Cell Physiol Biochem 2016;38:469-486.

18 Shankar J, Messenberg A, Chan J, Underhill TM, Foster LJ, Nabi IR: Pseudopodial actin dynamics control epithelial-mesenchymal transition in metastatic cancer cells. Cancer Res 2010;70:3780-3790.

19 Sobierajska K, Skurzynski S, Stasiak M, Kryczka J, Cierniewski CS, Swiatkowska M: Protein disulfide isomerase directly interacts with $\beta$-actin Cys374 and regulates cytoskeleton reorganization. J Biol Chem 2014;289:5758-5773.

20 Han X, Tian Y, Tian D: Tumor metastatic promoter ABCE1 interacts with the cytoskeleton protein actin and increases cell motility. Oncol Rep 2016;35:3623-3629.

21 Cai M, Wang Z, Zhang J, Zhou H, Jin L, Bai R, Weng Y: Adam17, a Target of Mir-326, Promotes Emt-Induced Cells Invasion in Lung Adenocarcinoma. Cell Physiol Biochem 2015;36:1175-1185. 


\section{Cellular Physiology Cell Physiol Biochem 2017;44:554-566 \begin{tabular}{l|l|l|l} 
DOI: 10.1159/000485090 & O 2017 The Author(s). Published by S. Karger AG, Basel \\
mww.karger.com/cpb
\end{tabular} \\ Published online: November 17,}

-22 Yang ZT, Li Z, Wang XG, Tan T, Yi F, Zhu H, Zhao JP, Zhou XF: Overexpression of Long Non-Coding RNA ZXF2 Promotes Lung Adenocarcinoma Progression Through c-Myc Pathway. Cell Physiol Biochem 2015;35:23602370.

23 Young DJ, Guydosh NR, Zhang F, Hinnebusch AG, Green R: Rli1/ABCE1 recycles terminating ribosomes and controls translation reinitiation in 3'UTRs invivo. Cell 2015;162:872-884.

24 Robinson BA, Reed JC, Geary CD, Swain JV, Lingappa JR: A temporospatial map that defines specific steps at which critical surfaces in the Gag MA and CA domains act during immature HIV-1 capsid assembly in cells. J Virol 2014;88:5718-5741.

25 Zhai C, Li Y, Mascarenhas C, Lin Q Li K, Vyrides I, Grant CM, Panaretou B: The function of ORAOV1/LTO1, a gene that is overexpressed frequently in cancer: Essential roles in the function and biogenesis of the ribosome. Oncogene 2014;33:484-494.

26 Olson MF, Sahai E: The actin cytoskeleton in cancer cell motility. Clin Exp Metastasis 2009;26:273-287.

27 Simiczyjew A, Mazur AJ, Dratkiewicz E, Nowak D: Involvement of $\beta$ - and $\gamma$-actin isoforms in actin cytoskeleton organization and migration abilities of bleb-forming human colon cancer cells. PLOS One 2017;12:e0173709.

28 Shagieva GS, Domnina LV, Chipysheva TA, Ermilova VD, Chaponnier C, Dugina VB: Actin isoforms and reorganization of adhesion junctions in epithelial-to-mesenchymal transition of cervical carcinoma cells. Biochemistry (Mosc) 2012;77:1266-1276.

29 Sims LM, Igarashi RY: Regulation of the ATPase activity of ABCE1 from Pyrococcus abyssi by Fe-S cluster status and $\mathrm{Mg}^{2+}$ : Implication for ribosomal function. Arch Biochem Biophys 2012;524:114-122.

-30 Hu P, Li Y, Sands MS, McCown T, Kafri T: Generation of a stable packaging cell line producing high-titer PPTdeleted integration-deficient lentiviral vectors. Mol Ther Methods Clin Dev 2015;2:15025.

-31 Kim SH, Jun HJ, Jang SI, You JC: The determination of importance of sequences neighboring the Psi sequence in lentiviral vector transduction and packaging efficiency. PLOS One 2012;7:e50148.

-32 Kroon J, in 't Veld LS, Buijs JT, Cheung H, van der Horst G, van der Pluijm G: Glycogen synthase kinase-3 $\beta$ inhibition depletes the population of prostate cancer stem/progenitor-like cells and attenuates metastatic growth. Oncotarget 2014;5:8986-8994.

-33 Ridley AJ, Schwartz MA, Burridge K, Firtel RA, Ginsberg MH, Borisy G, Parsons JT, Horwitz AR: Cell migration: Integrating signals from front to back. Science 2003;302:1704-1709.

34 Visegrády B, Lorinczy D, Hild G, Somogyi B, Nyitrai M: A simple model for the cooperative stabilisation of actin filaments by phalloidin and jasplakinolide. FEBS Lett 2005;579:6-10. 